\title{
STUDY ON PROPAGATION REGULARITY OF HARMONIC WAVES IN PERIODIC STRUCTURES OF BEAMS
}

\author{
Volodymyr Bulgakov ${ }^{1}$, Anastasiya Kutsenko ${ }^{1}$, Semjons Ivanovs ${ }^{2}$, Simone Pascuzzi $^{3}$ \\ ${ }^{1}$ National University of Life and Environmental Sciences of Ukraine, Ukraine; \\ ${ }^{2}$ Latvia University of Life Sciences and Technologies, Latvia; ${ }^{3}$ University of Bari Aldo Moro, Italy \\ semjons@apollo.lv, simone.pascuzzi@uniba.it
}

\begin{abstract}
The wide application of elements with periodic configuration in dynamically loaded structures stimulates a study of the propagation phenomenon of harmonic waves in mechanical systems of a periodic structure. The absolute majority of investigations of the wave propagation phenomenon in periodic structures concern one-dimensional and quasi-one-dimensional systems. Therefore, it is of certain interest to study the regularities in the distribution of the "transparency windows" of a mechanical system of a periodic structure with continuously changing parameters, which can be obtained by passing through the limit from the corresponding system with the threshold-type irregularities. Besides, the non-homogeneity in these systems is of a discrete, i.e. threshold type. The article presents the research results of propagation of harmonic waves along unfixed beams of a periodic structure. In addition two beam configurations are considered: with a constant cross section in individual segments and a continuous change in the cross section along the beam axis. The solution of the two problems has been made on the basis of the Floquet theory. As a result of the solution of this problem, frequency bands of the waves for both types of beams were found, and a comparative analysis of their propagation was carried out.
\end{abstract}

Key words: harmonic waves, frequency bands, "transparency windows".

\section{Introduction}

The wide application of elements with periodic configuration in dynamically loaded structures stimulates a study of the propagation phenomenon of harmonic waves in mechanical systems of a periodic structure. The results of these studies are used for the development of a variety of mechanical devices, including agricultural machinery [1], soundproofing systems for aircraft [2], mirrors for adaptive optics [3], etc.

Most studies of the wave propagation regularities in the systems of periodic structure are based on the Floquet theory [4], according to which all the kinematic and dynamic characteristics at similar points of any two neighbouring periods of the system differ $S$ times. Parameter $S$, which is usually called a multiplier, is generally a complex value:

$$
S=|S| e^{i \arg S} .
$$

The multiplier module indicates the diminution degree of the wave amplitude from period to period and the argument for the phase shift. It is obvious that $|S|=1$ corresponds to the frequency intervals of the passage of waves, which we will call "transparency windows". The discovery of "transparency windows" is the main goal in studying the fluctuations of the periodic structures.

It should be noted that the absolute majority of investigations of the wave propagation phenomenon in periodic structures concern one-dimensional and quasi-one-dimensional systems. Besides, the non-homogeneity in these systems is of a discrete, i.e. threshold type. As an example of periodic systems of the threshold type homogeneous beams or beams of a stepped cross section (with separate sections of the constant section) periodically fixed at individual points can serve [5]. Therefore, it is of certain interest to study the regularities in the distribution of the "transparency windows" of a mechanical system of a periodic structure with continuously changing parameters, which can be obtained by passing through the limit from the corresponding system with the thresholdtype irregularities. In this paper similar studies have been conducted on a beam of variable cross section as an example.

\section{Materials and methods}

To achieve this goal, it is interesting to compare the distribution of the "transparency windows" for a system with threshold non-homogeneity and for a system that can be obtained from the first one by limit transition. Therefore, on the basis of the Floquet theory, it is necessary to solve two tasks, respectively. The first task is to determine the "transparency windows" of the beam, each half-period 
of which consists of $N$ homogeneous parts (that is, the number of parts of a homogeneous section). One period of such a beam in Fig. 1 at $N=3$ is shown in gray.

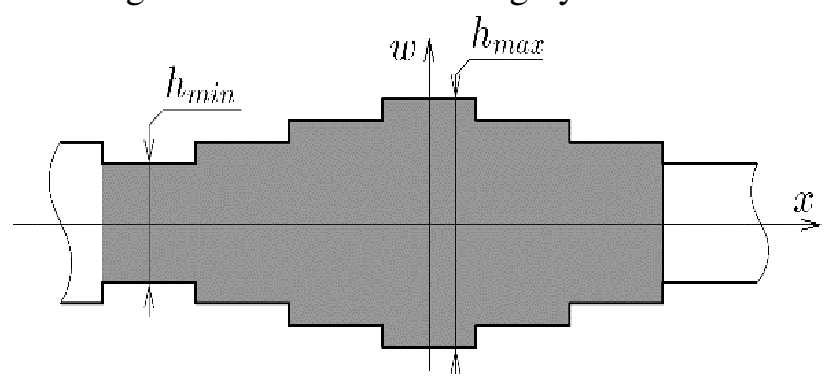

Fig. 1. Period of beam of stepped cross section (side view)

Let us assume that the width of the beam $b$ does not change along the axis $x$ of the beam, and the length of each section, on which the height of the beam is constant, is

$$
a_{0}=\frac{a}{2 N},
$$

where $a$-length of the period of the beam.

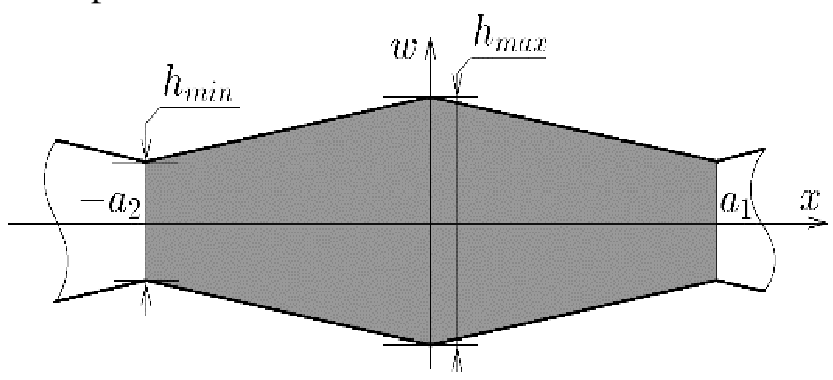

Fig. 2. Period of beam with continuously changing cross-section (side view)

The second task is to determine the "transparency windows" of the beam, which can be obtained from the first by passing through the limit (limit transition) $N \rightarrow \infty$. The period of the second beam is shown in Fig. 2 in grey. We will also assume that the width of the beam $b$ does not change along the axis $x$ of the beam, and then we have:

$$
a_{1}=a_{2}=\frac{a}{2}
$$

where $a_{1}$ - length of a half-period of the beam.

Since the formulated problems are one-dimensional, and the dependence of all characteristics on time $t$ is harmonic, the solution will be based on analytical methods. Assuming that the deflection $w(x, t)$ of each of the beams can be represented in the form $w(x, t)=w(x) e^{i \omega t}$ relative to the amplitude function of the deflections $w(x)$ in one period, we obtain a linear equation [6],

$$
\frac{d^{2}}{d x^{2}}\left[j(x) \frac{d^{2} w(x)}{d x^{2}}\right]-p^{4} w(x)=0,|x|<a / 2,
$$

where $j(x)=\frac{J(x)}{J_{\min }}, J(x)=\frac{b h^{3}(x)}{12}-$ moment of inertia of the cross section of the beam;

$J_{\min }-$ minimal value of the moment of inertia;

$h(x)$ - height of the beam, $p=\sqrt{\omega \sqrt{\frac{\rho F(x)}{E J_{\text {min }}}}} ;$

$F(x)=b h(x)-$ cross section area of the beam;

$\rho, E$ - density and the modulus of elasticity of the beam material;

$\omega-$ circular fluctuation frequency. 
We also note [6] the differential connection between the amplitude functions of the deflections, turning angles to the cross section $\vartheta(x)$, the bending moments $M(x)$ and the shear forces $Q(x)$ :

$$
\begin{gathered}
\vartheta(x)=\frac{d w(x)}{d x}, \\
M(x)=E J(x) \frac{d^{2} w(x)}{d x^{2}}, \\
Q(x)=\frac{d}{d x}\left[E J(x) \frac{d^{2} w(x)}{d x^{2}}\right] .
\end{gathered}
$$

In order to obtain a solution to the problem, i.e. dependence of the multiplier on the dimensionless frequency $p a$, it is necessary to subject the general solution of equation (1) to the conditions of a quasiperiodical extension:

$$
\begin{aligned}
& w\left(\frac{a}{2}\right)=S w\left(-\frac{a}{2}\right), \\
& \vartheta\left(\frac{a}{2}\right)=S \vartheta\left(-\frac{a}{2}\right), \\
& M\left(\frac{a}{2}\right)=S M\left(-\frac{a}{2}\right), \\
& Q\left(\frac{a}{2}\right)=S Q\left(-\frac{a}{2}\right)
\end{aligned}
$$

and to equate to zero the determinant of the obtained homogeneous system.

The proposed scheme is universal and can be used to solve the problem for a beam with arbitrary variation of the cross section. However, for a beam of a stepped cross section, which corresponds to the first task, it is more rational to determine the solution separately in each homogeneity interval, equation (1) being transformed into a differential equation with constant coefficients:

$$
\begin{gathered}
\frac{d^{4} w_{n}(x)}{d x^{4}}-p_{n}^{4} w_{n}(x)=0, \\
x<a_{0}, n=\overline{1,2 N},
\end{gathered}
$$

where $w_{n}(x)$-amplitude function of the deflections in interval $n$;

$$
p_{n}=\sqrt{\omega \sqrt{\frac{\rho F_{n}}{E J_{n}}}}=\text { const } .
$$

The general solution of equation (4) has a simple form:

$$
w_{n}(x)=A_{n} \cos \left(p_{n} x\right)+B_{n} \sin \left(p_{n} x\right)+C_{n} \operatorname{ch}\left(p_{n} x\right)+D_{n} \operatorname{sh}\left(p_{n} x\right),
$$

where $A_{n}, B_{n}, C_{n}$ and $D_{n}$ - constants of integration.

Taking into account the differential correlations (3), solution (5) must be subjected to the conditions of uninterrupted continuation at the junction points of the sections:

$$
\begin{gathered}
w_{n}\left(a_{0}\right)=w_{n+1}(0), \\
\vartheta_{n}\left(a_{0}\right)=\vartheta_{n+1}(0), \\
M_{n}\left(a_{0}\right)=M_{n+1}(0),
\end{gathered}
$$




$$
\begin{gathered}
Q_{n}\left(a_{0}\right)=Q_{n+1}(0), \\
n=\overline{1,2 N-1} .
\end{gathered}
$$

In this case, the conditions for the quasi-periodic continuation (3) assume the form:

$$
\begin{gathered}
w_{2 N}\left(a_{0}\right)=S w_{1}(0), \\
\vartheta_{2 N}\left(a_{0}\right)=S \vartheta_{1}(0), \\
M_{2 N}\left(a_{0}\right)=S M_{1}(0), \\
Q_{2 N}\left(a_{0}\right)=S Q_{1}(0) .
\end{gathered}
$$

Consequently, on the basis of correlations (2) and (5)-(7) we obtain a system of homogeneous equations. By equating its determinant to zero, we obtain an algebraic equation of the fourth power with respect to the multiplier. The solution of the latter does not present fundamental difficulties, since for physical considerations one can maintain that, alongside with a certain root $S$ of this equation, its root will be also the inverse value $1 / S$.

When discussing the problem for the second beam, each period of which consists of two wedgeshaped parts, we will proceed directly from equation (1) that, taking into account the concrete dependence $h=h(x)$, is represented as:

$$
\begin{gathered}
\frac{d^{2}}{d x^{2}}\left[\left(1+2 \alpha \frac{|x|}{a}\right)^{3} \frac{d^{2} w(x)}{d x^{2}}\right]-\left(1+2 \alpha \frac{|x|}{a}\right) p^{4} w(x)=0, \\
|x|<\frac{a}{2},
\end{gathered}
$$

where $\quad \alpha=\frac{h_{\max }}{h_{\min }}-1-$ thickness difference of the beam.

The search for the solution of equation (8) was made by Kirchhoff [7]. He succeeded in obtaining some of the first terms of the asymptotic expansion of the solution for small angles of the wedge divergence. In a closed form the solution of equation (8) was obtained by A. Dinnik [8;9].

For a wedge corresponding to the right side of the period $(x>0)$, it can be presented in this form:

$$
\begin{aligned}
& w(x)=A \frac{J_{1}\left(2 \frac{p a}{\alpha} \sqrt{1+2 \alpha \frac{x}{a}}\right)}{\sqrt{1+\frac{2 \alpha x}{a}}}+B \frac{Y_{1}\left(2 \frac{p a}{\alpha} \sqrt{1+2 \alpha \frac{x}{a}}\right)}{\sqrt{1+\frac{2 \alpha x}{a}}}+ \\
& +C \frac{I_{1}\left(2 \frac{p a}{\alpha} \sqrt{1+2 \alpha \frac{x}{a}}\right)}{\sqrt{1+\frac{2 \alpha x}{a}}}+A \frac{K_{1}\left(2 \frac{p a}{\alpha} \sqrt{1+2 \alpha \frac{x}{a}}\right)}{\sqrt{1+\frac{2 \alpha x}{a}}},
\end{aligned}
$$

where $J_{1}, Y_{1}-$ Bessel functions of the first and second kind of the first order,

$I_{1}, K_{1}-$ modified Bessel functions of the first and second kind of the first order,

$B, C$ and $D$ - constants of integration.

For a left wedge the expression for the deflection has a similar form, with accuracy up to the sign at $x$. After the conditions for uninterrupted continuation point $x=0$ and conditions of the quasiperiodic extension (3) are satisfied, we arrive at a homogeneous system of linear equations, the determinant of which is a polynomial of the $4^{\text {th }}$ degree with respect to the multiplier. Thus, the course 
of further solution of the second task completely coincides with the course of the solution of the first task.

\section{Results and discussion}

In a graphic form the results of solving the tasks are presented in Figures 3-6 that show (in gray) the "transparency windows" depending on the dimensionless frequency $p a$ and parameter $\alpha$ for a different number of sections of constancy of the cross section for a half-period $N$.

The first three distributions of the "transparency windows" (Figures 3-5) are obtained as a result of solving the first task, and the last distribution (Figure 6) is the result of solving the second task. When analysing the presented distributions, first of all, we should pay attention to the existence of passing the limit (limit transition) at $N \rightarrow \infty$.

Common for all diagrams is also the monotonous increase in the lengths of the intervals of the limit intransitivity from the zero value at $\alpha=0$ at point $n \pi$. Besides, the smaller is $N$, the more rapid is the increase in the lengths of the intransitivity intervals with the growth of $\alpha$, which fully corresponds to the physical foundations of the theory of wave propagation.

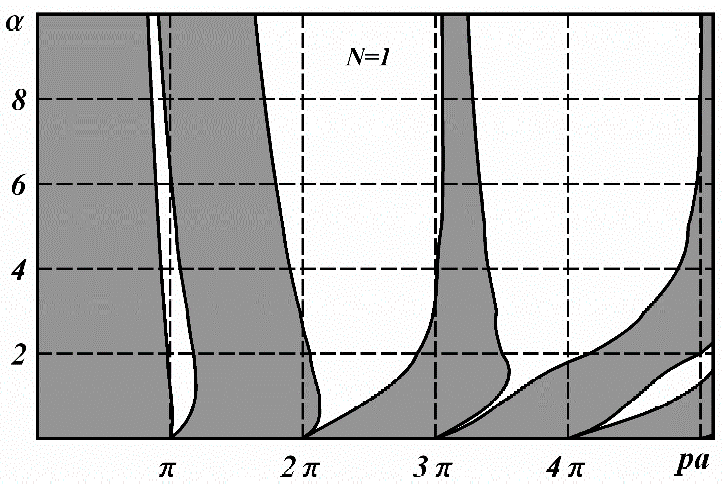

Fig. 3. "Transparency windows" of beam with one homogeneous section for half-period

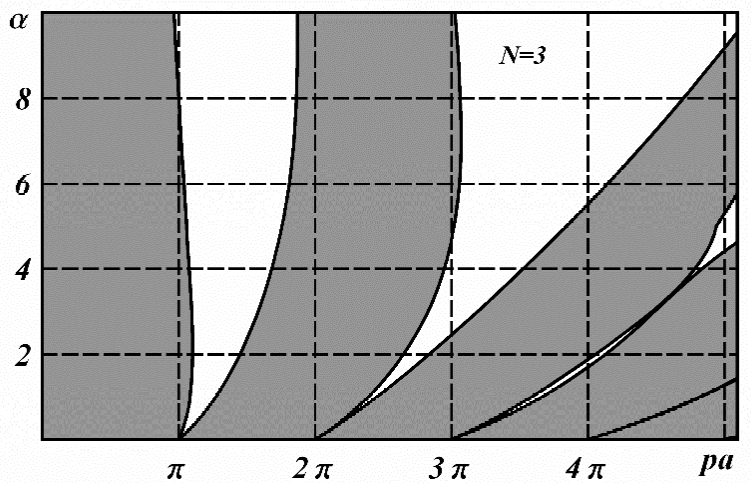

Fig. 4. "Transparency windows" of beam with three homogeneous sections for half-period

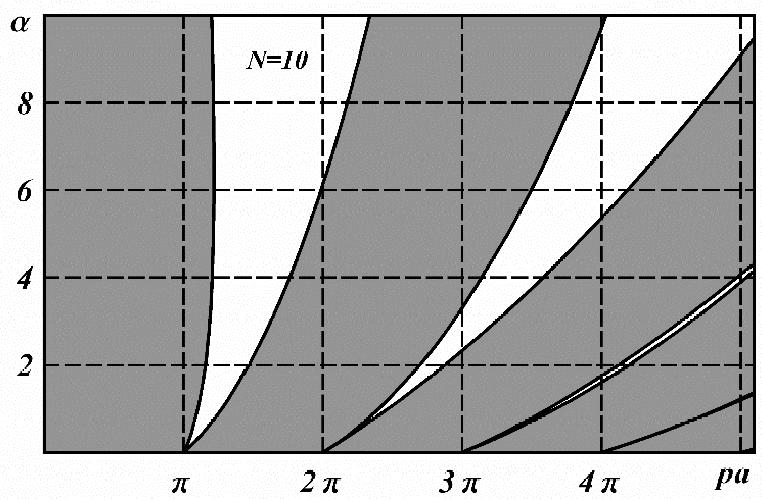

Fig. 5. "Transparency windows" of beam with ten homogeneous sections for half-period 
The boundary lines of the "windows of transparency", already depicted in Fig. 3-4, are bent with the increase in the thickness difference $\alpha$, collapsing to the left. This peculiarity is clearly visible at $N=1$ and $N=3$, but at careful examination it can be seen also for $N=10$. For this purpose attention is paid to the right border of the left "window" in Fig. 5. This is practically the only qualitative difference between Figures 5 and 6 . The consequence of bending the boundary lines is their intersection, as it is the case of Fig. 4 for the lines emerging from point $3 \pi$ at $\alpha=0$.

Another difference between the diagrams shown in Fig. 3-5 and in Fig. 6 is that in the first three figures the "windows" become narrower with the value of parameter $\alpha$ increasing.

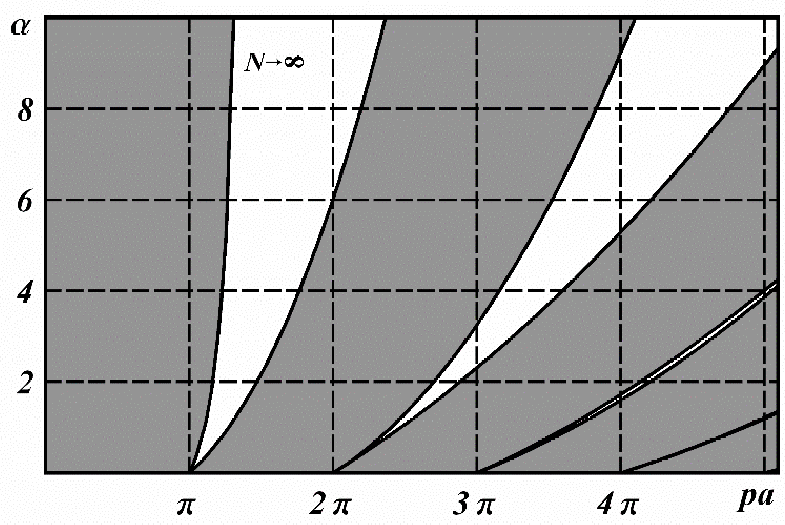

Fig. 6. "Transparency windows" of beam with wedge-shaped half-period

\section{Conclusions}

1. The "transparency windows" for a periodically structured beam with a stepwise cross section demonstrate the presence of a limit transition at $N \rightarrow \infty$, where $N$ is the number of constancy sections of the cross section for a half-period. In other words, the limit transition occurs when passing to a piecewise wedge-shaped beam.

2. The intervals between the "transparency windows" increase with an increase in the heterogeneity (the increase in $\alpha$ ) for any configuration of the beam.

3. In contrast to the case of a piecewise wedge-shaped beam, the boundaries of the "transparency windows" of the stepped beams collapse to the left with an increase in the non-uniformity of the beam.

4. In contrast to the case of a piecewise-wedge-shaped beam, the "windows of transparency" of the beams of the stepped section become narrower with the increase in the non-uniformity of the beam.

\section{References}

[1] Mead D.J. Wave propagation in continuous periodic structures: research contributions from Southampton, 1964-1995. J. Sound and Vibr., vol. 190, No 3, 1996, pp. 495-524.

[2] Clarkson B.L., Ford R.D. The response of a typical aircraft structure to jet noise J. Roy. Aeronaut. Soc., 1962, vol. 66, No 613, pp. 31-40.

[3] Tyson R.K. Principles of Adaptive Optics. Boston, Academic Press, 1998, 345 p.

[4] Brilloun L., Parodi M. Wave Propagation in Periodic Structures. Dover Publications, 2012, 457 p.

[5] Kucenko A. Propagation of waves in beams fixed in a periodic manner. Bulletin of the University of Kiev. A series of physical and mathematical sciences, 1997, No 3, pp. 69-76. (In Ukrainian).

[6] Bansal R.K. A Textbook of Strength of Materials, New Delhi, 2010, 1106 p.

[7] Todhunter I. A history of the theory of elasticity and of the strength of materials, vol. 2, part 2. Adamant Media Corporation, 2003, 566 p.

[8] Filippov A.P. Oscillations of mechanical systems. Scientific thought, Kiev, 1995, 716 p. (In Russian).

[9] Wolfson I. Oscillations in machines with the mechanisms of cyclic action. St. Petersburg, 2008. 260 p. (In Russian). 